NASA/TP—1999-209424/REV1

\title{
Generalized Functions for the Fractional Calculus
}

Carl F. Lorenzo

Glenn Research Center, Cleveland, Ohio

Tom T. Hartley

The University of Akron, Akron, Ohio 
Since its founding, NASA has been dedicated to the advancement of aeronautics and space science. The NASA Scientific and Technical Information (STI) Program Office plays a key part in helping NASA maintain this important role.

The NASA STI Program Office is operated by Langley Research Center, the Lead Center for NASA's scientific and technical information. The NASA STI Program Office provides access to the NASA STI Database, the largest collection of aeronautical and space science STI in the world. The Program Office is also NASA's institutional mechanism for disseminating the results of its research and development activities. These results are published by NASA in the NASA STI Report Series, which includes the following report types:

- TECHNICAL PUBLICATION. Reports of completed research or a major significant phase of research that present the results of NASA programs and include extensive data or theoretical analysis. Includes compilations of significant scientific and technical data and information deemed to be of continuing reference value. NASA's counterpart of peerreviewed formal professional papers but has less stringent limitations on manuscript length and extent of graphic presentations.

- TECHNICAL MEMORANDUM. Scientific and technical findings that are preliminary or of specialized interest, e.g., quick release reports, working papers, and bibliographies that contain minimal annotation. Does not contain extensive analysis.

- CONTRACTOR REPORT. Scientific and technical findings by NASA-sponsored contractors and grantees.
- CONFERENCE PUBLICATION. Collected papers from scientific and technical conferences, symposia, seminars, or other meetings sponsored or cosponsored by NASA.

- SPECIAL PUBLICATION. Scientific, technical, or historical information from NASA programs, projects, and missions, often concerned with subjects having substantial public interest.

- TECHNICAL TRANSLATION. Englishlanguage translations of foreign scientific and technical material pertinent to NASA's mission.

Specialized services that complement the STI Program Office's diverse offerings include creating custom thesauri, building customized data bases, organizing and publishing research results ... even providing videos.

For more information about the NASA STI Program Office, see the following:

- Access the NASA STI Program Home Page at http://www.sti.nasa.gov

- E-mail your question via the Internet to help@sti.nasa.gov

- Fax your question to the NASA Access Help Desk at (301) 621-0134

- Telephone the NASA Access Help Desk at (301) 621-0390

- Write to:

NASA Access Help Desk

NASA Center for AeroSpace Information

7121 Standard Drive

Hanover, MD 21076 
NASA/TP-1999-209424/REV1

\section{Generalized Functions for the Fractional Calculus}

Carl F. Lorenzo

Glenn Research Center, Cleveland, Ohio

Tom T. Hartley

The University of Akron, Akron, Ohio

National Aeronautics and

Space Administration

Glenn Research Center 
This copy replaces all previous copies printed of this report. Print errors have been corrected.

Available from

NASA Center for Aerospace Information 7121 Standard Drive

Hanover, MD 21076

Price Code: A03
National Technical Information Service 5285 Port Royal Road Springfield, VA 22100 Price Code: A03 


\title{
Generalized Functions for the Fractional Calculus
}

\author{
Carl F. Lorenzo \\ National Aeronautics and Space Administration \\ Glenn Research Center \\ Cleveland, Ohio 44135 \\ Tom T. Hartley \\ The University of Akron \\ Department of Electrical Engineering \\ Akron, Ohio 44325-3904
}

\section{Introduction}

Previous papers have used two important functions for the solution of fractional order differential equations, the Mittag-Leffler function $E_{4}\left[a t^{q}\right](1903 \mathrm{a}, 1903 \mathrm{~b}, 1905)$, and the F-function $F_{q}[a, t]$ of Hartley \& Lorenzo (1998). These functions provided direct solution and important understanding for the fundamental linear fractional order differential equation and for the related initial value problem (Hartley and Lorenzo, 1999).

This paper examines related functions and their Laplace transforms. Presented for consideration are two generalized functions, the $R$-function and the $G$-function, useful in analysis and as a basis for computation in the fractional calculus. The $R$-function is unique in that it contains all of the derivatives and integrals of the F-function. The $R$-function also returns itself on $q$ th order differ-integration. An example application of the $R$-function is provided. A further generalization of the $R$-function, called the $G$-function brings in the effects of repeated and partially repeated fractional poles.

\section{Functions for the Fractional Calculus}

This section summarizes a number of functions that have been found useful in the solution of problems of the fractional calculus and more particularly in the solution of fractional differential equations.

\section{Mittag-Leffler Function}

The Mittag-Leffler $(1903,1903,1905)$ function is given by the following equation

$$
E_{q}[t]=\sum_{n=0}^{\infty} \frac{t^{n}}{\Gamma(n q+1)}, \quad q>0 .
$$

This function will often appear with the argument $-a t^{q}$, its Laplace transform then, is given as

$$
L\left\{E_{q}\left[-a t^{q}\right]\right\}=L\left\{\sum_{n=0}^{\infty} \frac{(-a)^{n} t^{n q}}{\Gamma(n q+1)}\right\}=\frac{s^{q}}{s\left(s^{q}+a\right)} \quad q>0 .
$$




\section{Agarwal's Function}

The Mittag-Leffler function is generalized by Agarwal (1953) as follows

$$
E_{\alpha, \beta}(t)=\sum_{m=0}^{\infty} \frac{t^{\left(m+\frac{\beta-1}{\alpha}\right)}}{\Gamma(\alpha m+\beta)} .
$$

This function is particularly interesting to the fractional order system theory due to its Laplace transform, given by Agarwal as

$$
L\left\{E_{\alpha, \beta}\left[t^{\alpha}\right]\right\}=\frac{s^{\alpha-\beta}}{s^{\alpha}-1}
$$

This function is the $(\alpha-\beta)$ order fractional derivative of the F-function, (of Robotnov (1969) and Hartley (1998)), with argument $a=1$, to be presented later.

\section{Erdelyi's Function}

Erdelyi (1954) has studied the following related generalization of the Mittag-Leffler function

$$
E_{\alpha, \beta}(t)=\sum_{m=0}^{\infty} \frac{t^{m}}{\Gamma(\alpha m+\beta)}, \quad \alpha, \beta>0,
$$

where the powers of $t$ are integer. The Laplace transform of this function is given by

$$
L\left\{E_{\alpha, \beta}(t)\right\}=\sum_{m=0}^{\infty} \frac{\Gamma(m+1)}{\Gamma(\alpha m+\beta) s^{m+1}} \quad \alpha, \beta>0 .
$$

As this function cannot be easily generalized it will not be considered further.

\section{Robotnov and Hartley's Function}

To effect the direct solution of the fundamental linear fractional order differential equation the following function was introduced (Hartley and Lorenzo, 1998)

$$
F_{q}[-a, t]=t^{q-1} \sum_{n=0}^{\infty} \frac{(-a)^{n} t^{n q}}{\Gamma(n q+q)} \quad q>0 .
$$

This function had been studied earlier by Robotnov $(1969,1980)$ with respect to hereditary integrals for application to solid mechanics. The important feature of this function is the power and simplicity of its Laplace transform, namely

$$
L\left\{F_{q}[a, t]\right\}=\frac{1}{s^{q}-a}, \quad q>0 .
$$

\section{Miller and Ross' Function}

Miller and Ross (1993, pp.80 and 309-351) introduce another function as the basis of the solution of the fractional order initial value problem. It is defined as the $v$ th integral of the exponential function, that is

$$
E_{t}(v, a)=\frac{d^{-v}}{d t^{-v}} e^{a t}=t^{v} e^{a t} \gamma^{*}(v, a t)=t^{\prime} \sum_{k=0}^{\infty} \frac{(a t)^{k}}{\Gamma(v+k+1)},
$$


where $\gamma^{*}(v, a t)$ is the incomplete gamma function. The Laplace transform of equation (9) follows directly as

$$
L\left\{E_{t}(v, a)\right\}=\frac{s^{-v}}{s-a} \quad \operatorname{Re}(v)>1
$$

Miller and Ross then show that

$$
L\left\{\sum_{j=1}^{q} a^{j-1} E_{t}\left(j v-1, a^{q}\right)\right\}=\frac{1}{s^{r}-a}, q=1,2,3 \ldots, v=\frac{1}{q}=1, \frac{1}{2}, \frac{1}{3}, \ldots,
$$

which is a special case of the F-function of Robotnov and Hartley.

The above functions are studied in considerable detail by their originators and others. The interested reader is directed to the supplied references.

\section{A Generalized Function}

It is of significant usefulness to develop a generalized function which when fractionally differintegrated (by any order) returns itself. Such a function would greatly ease the analysis of fractional order differential equations. To this end the following is proposed, consider the function

$$
R_{q, v}[a, c, t] \equiv \sum_{n=0}^{\infty} \frac{(a)^{n}(t-c)^{(n+1) q-1-v}}{\Gamma((n+1) q-v)}
$$

Our interest in this function will normally be for the solution of fractional differential equations for the range of $t>c=0$. For $t<c, R$ will be complex except for the cases when the exponent $((n+1) q-1-v)$ is integer. The more compact notation

$$
R_{q . v}[a, t-c]=\sum_{n=0}^{\infty} \frac{(a)^{n}(t-c)^{(n+1)_{q-1-v}}}{\Gamma((n+1) q-v)}
$$

is also useful, particularly when $c=0$.

The Laplace transform of the $R$-function is

$$
L\left\{R_{q, v}[a, c, t]\right\}=L \sum_{n=0}^{\infty} \frac{(a)^{n}(t-c)^{(n+1) q-1-v}}{\Gamma((n+1) q-v)}=\sum_{n=0}^{\infty}(a)^{n} L\left\{\frac{(t-c)^{(n+1)_{q-1-v}}}{\Gamma((n+1) q-v)}\right\} .
$$

Consider first the case for $c=0$, then we have

$$
L\left\{R_{q, v}[a, 0, t]\right\}=\sum_{n=0}^{\infty}(a)^{n} L\left\{\frac{(t)^{(n+1)_{q-1-v}}}{\Gamma((n+1) q-v)}\right\} .
$$

Now from (Erdelyi et al, 1954)

$$
L\left\{t^{v}\right\}=\Gamma(v+1) s^{-v-1} \quad \operatorname{Re}(v)>-1, \operatorname{Re}(s)>0 .
$$

Then equation 15 becomes

$$
L\left\{R_{q, v}[a, 0, t]\right\}=\sum_{n=0}^{\infty}(a)^{n} \frac{1}{s^{(n+1) q-v}} \quad \operatorname{Re}((n+1) q-v)>0, \operatorname{Re}(s)>0 .
$$




$$
L\left\{R_{q \cdot v}[a, 0, t]\right\}=\frac{1}{s^{-v}} \sum_{n=0}^{\infty} \frac{(a)^{n}}{s^{(n+1) q}} \quad \operatorname{Re}((n+1) q-v)>0, \operatorname{Re}(s)>0 .
$$

This can be written as a geometric series that converges when $\left|a / s^{4}\right|<1$. It can be shown, by long division, that

$$
L\left\{R_{q, v}(a, 0, t)\right\}=\frac{s^{v}}{s^{q}-a}, \quad \operatorname{Re}(q-v)>0, \quad \operatorname{Re}(s)>0 .
$$

Now for $c \neq 0$ the shifting theorem for the Laplace transform (Wylie p. 281) is

$$
L\{f(t-b) \mu(t-b)\}=e^{-b s} L\{f(t)\} \quad b \geq 0,
$$

where the unit step function $u(t-b)$ effectively causes $f(t-b)=0$ for $t<b$. Under the assumption that $R_{q, v}[a, c, t]=0$ for $t<c$, this theorem and the result (equation 19) are applied to yield

$$
L\left\{R_{q, v}(a, c, t)\right\}=\frac{e^{-c s} s^{v}}{s^{q}-a} \quad c \geq 0, \operatorname{Re}((n+1) q-v)>0, \operatorname{Re} s>0 .
$$

Table 1 , in a later section, presents a summary of the defining series and respective Laplace transforms for the functions discussed in this paper.

\section{Properties of the $R_{q, v}(a, c, t)$ Function}

The general time domain character of the $R$-function is shown in figures 1,2 , and 3 . Figure 1 shows the effect of variations in $q$ with $v=0$ and $a= \pm 1$. The exponential character of the function is readily observed (see, $q=1$ ). Figure 2 shows the effect of $v$ on the behavior of the $R$-function. The effect of the characteristic time $a$ is shown in figure 3 . The characteristic time is $1 / a^{q}$. For $q=1,1 / a$ is the time constant, when $q=2$ we have the natural frequency, when $q$ takes on other values we have the generalized characteristic time (or generalized time constant).

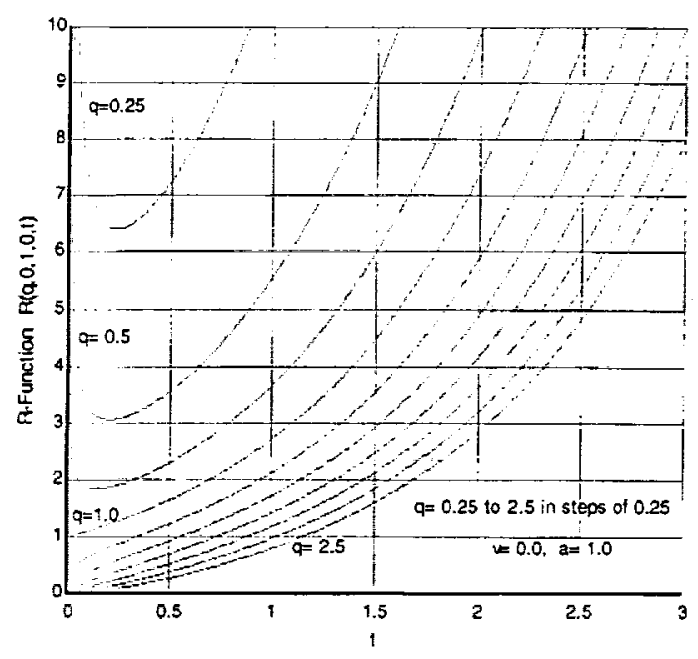

Figure 1a. Effect of $q$ on $R_{4,0}(1,0, t)$ $v=0.0, a=1.0$

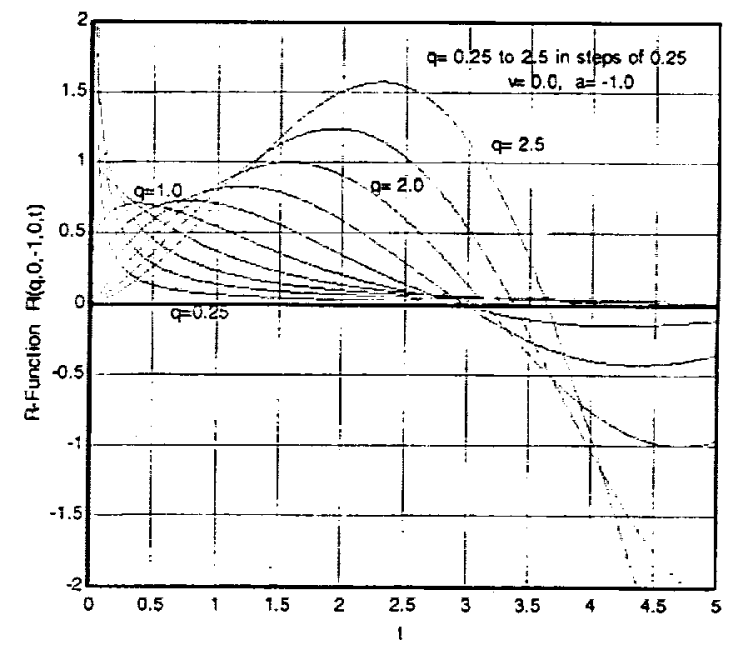

Figure 1b. Effect of $q$ on $R_{q, 0}(-1,0, t)$, $v=0.0, a=-1.0$ 


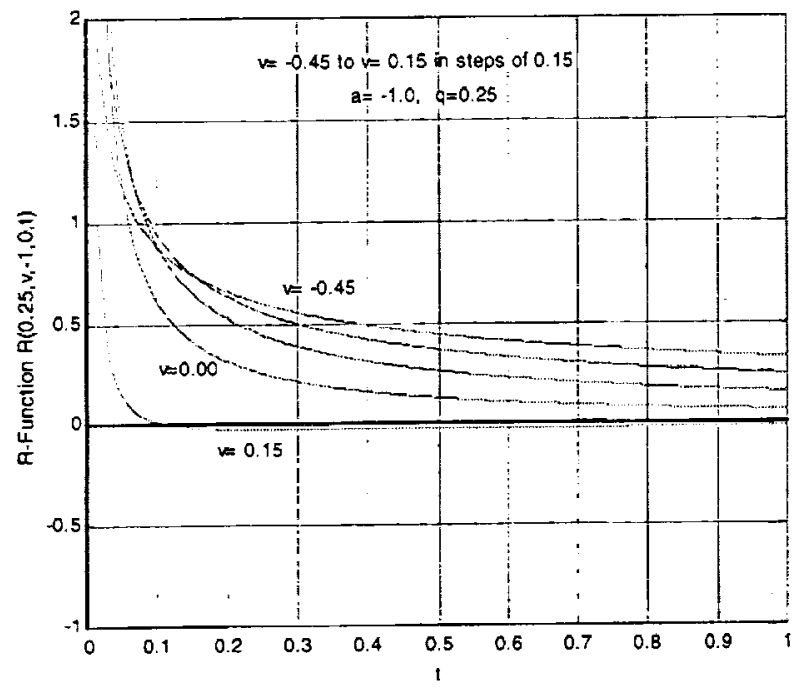

Figure 2a. Effect of $v$ on $R_{0.25, v}(-1,0, t)$ $q=0.25, a=-1.0$

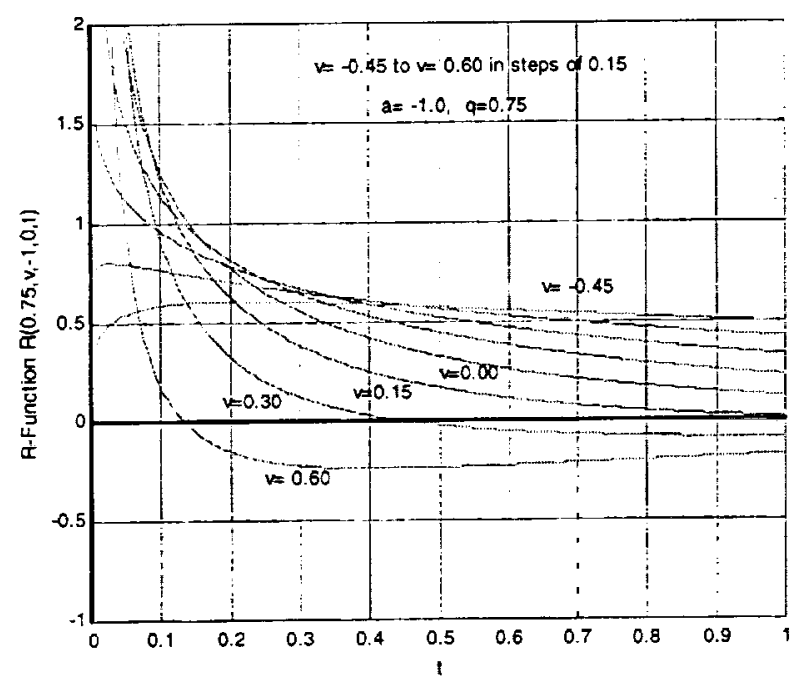

Figure 2c. Effect of $v$ on $R_{0.75, v}(-1,0, t)$ $q=0.75, a=-1.0$

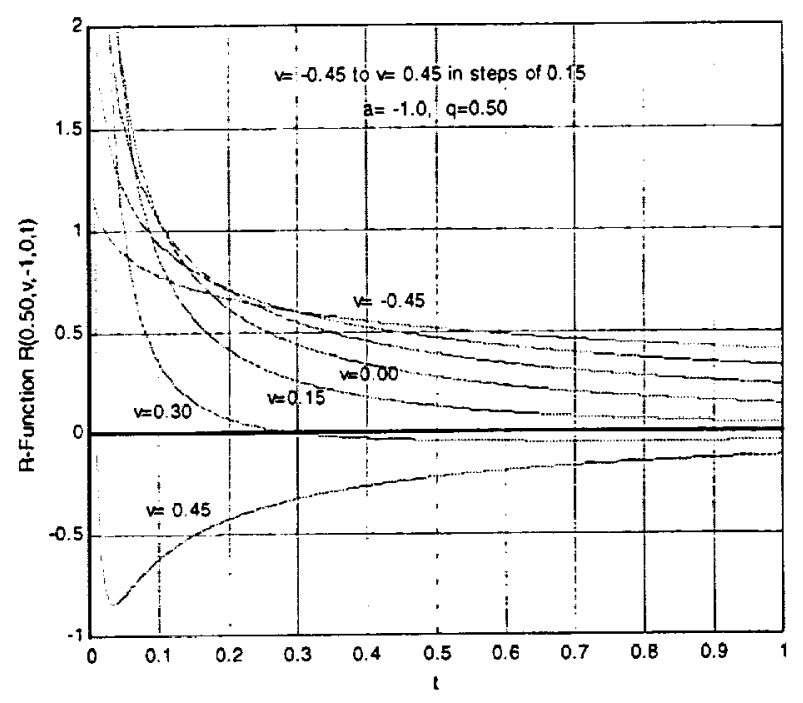

Figure $2 \mathrm{~b}$. Effect of $v$ on $R_{0.50, v}(-1,0, t)$ $q=0.50, a=-1.0$

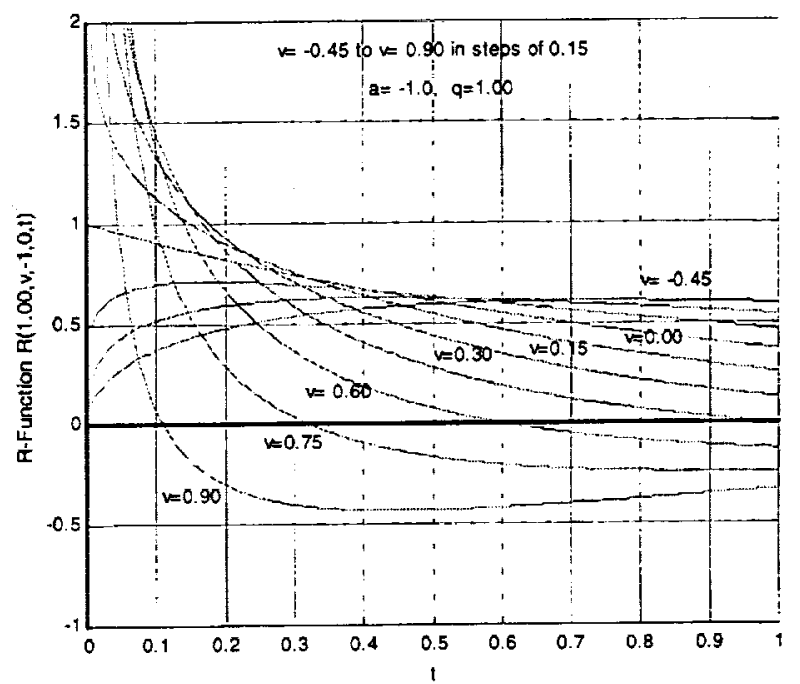

Figure 2d. Effect of $v$ on $R_{1.00, v}(-1,0, t)$ $q=1.00, a=-1.0$ 


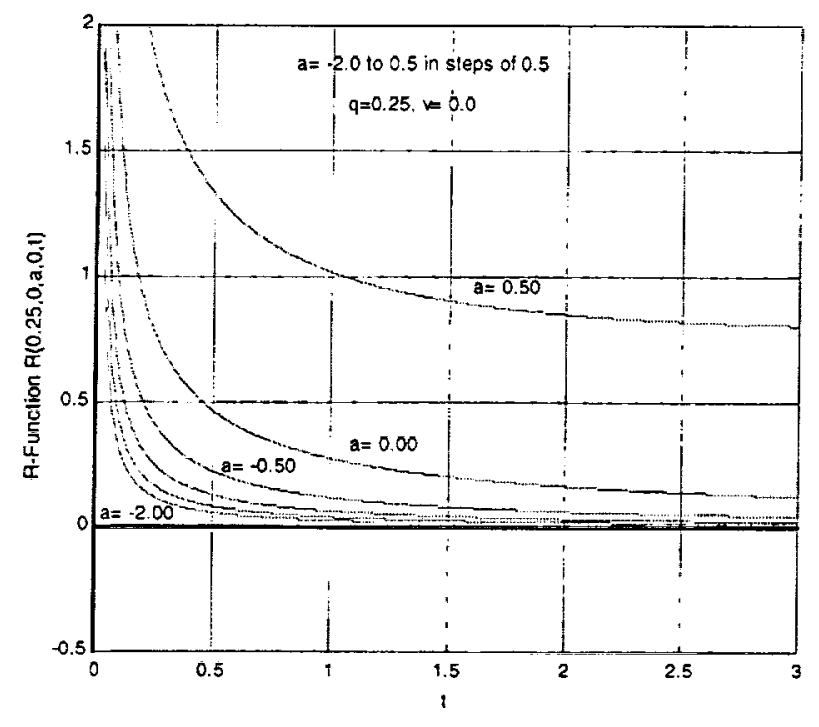

Figure 3a. Effect of $a$ on $R_{0.25,0}(a, 0, t)$ $q=1.00, v=0.0$

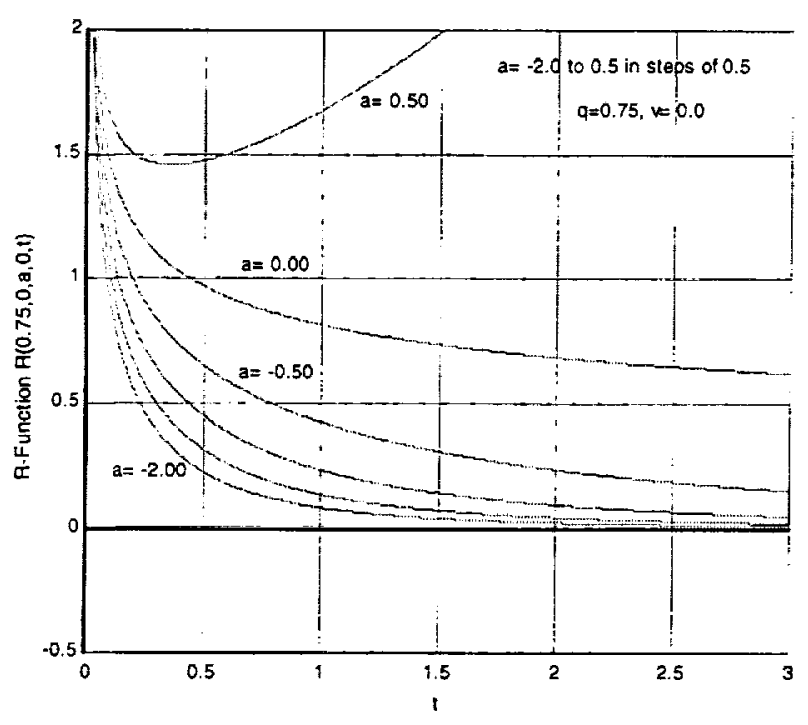

Figure $3 c$. Effect of $a$ on $R_{0.75,0}(a, 0, t)$ $q=0.75, v=0.0$

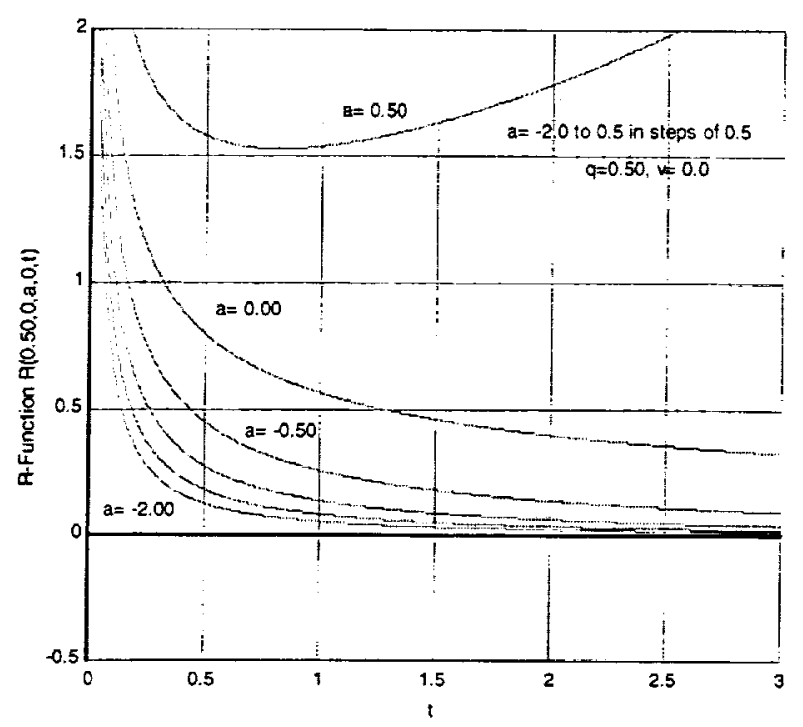

Figure 3b. Effect of $a$ on $R_{0,50,0}(a, 0, t)$ $q=0.50, v=0.0$

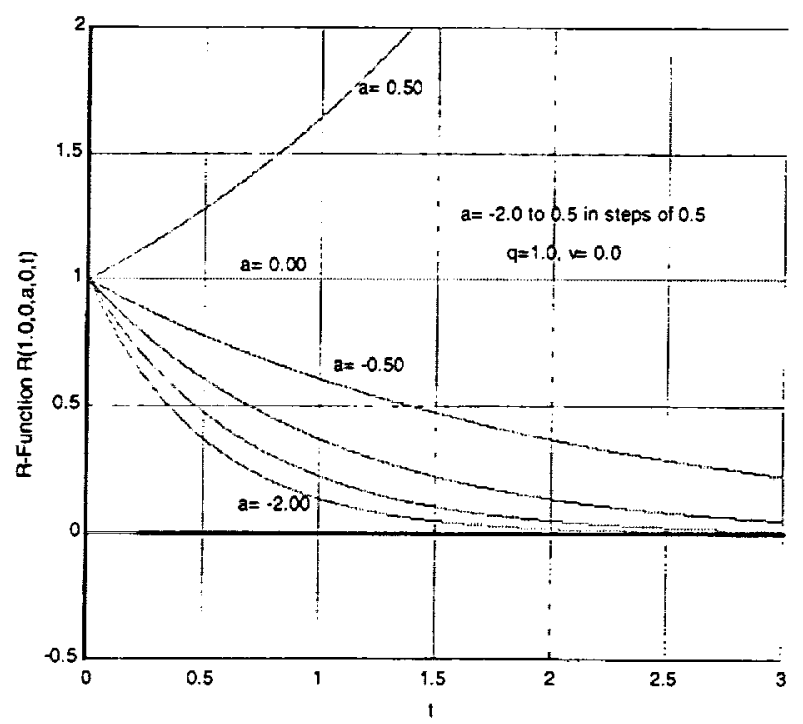

Figure 3d. Effect of $a$ on $R_{1.00,0}(a, 0, t)$ $q=1.00, v=0.0$ 


\section{Eigen-property}

The $R$-function also has the eigenfunction character under $q$ th order differintegration with $v=0$. This is seen as follows. Consider

$$
{ }_{c} d_{t}^{q} R_{q, 0}(a, c, t)=\sum_{n=0}^{\infty} \frac{(a)^{n}{ }_{c} d_{t}^{q}(t-c)^{(n+1)_{q-1}}}{\Gamma((n+1) q)} .
$$

Now, Oldham and Spanier (1974 p.67) prove the following useful form

$$
{ }_{a} d_{x}^{v}[x-a]^{p}=\frac{\Gamma(p+1)[x-a]^{p-v}}{\Gamma(p-v+1)} \quad p>-1
$$

Applying this equation we have

$$
{ }_{c} d_{t}^{q} R_{q, 0}(a, c, t)=\sum_{n=0}^{\infty} \frac{(a)^{n}(t-c)^{n q-1}}{\Gamma(n q)} \quad q>0 .
$$

Now let $n=m+1$, then,

$$
=\sum_{m=-1}^{\infty} \frac{(a)^{m+1}(t-c)^{(m+1) q-1}}{\Gamma((m+1) q)} \quad q>0
$$

or

$$
{ }_{c} d_{t}^{q} R_{q, 0}(a, c, t)=(a) R_{q, 0}(a, c, t)+a \lim _{m \rightarrow-1} \frac{(a)^{m}(t-c)^{(m+1)_{q-1}}}{\Gamma((m+1) q)} \quad q>0 .
$$

The right most term in equation (26) is zero for $t \neq c$, thus, for $t>c$ the final result is

$$
{ }_{c} d_{t}^{q} R_{q, 0}(a, c, t)=a R_{4,0}(a, c, t) \quad t>c, q>0 .
$$

Thus, for $a=1$ the function is seen to retum itself under $q$ th order differentiation.

\section{Differintegration of the $R$-Function}

It is of interest to determine the differintegral of the $R$-function, that is

$$
{ }_{c} d_{t}^{u} R_{q, v}[a, c, t] \models_{c} d_{t}^{u} \sum_{n=0}^{\infty} \frac{(a)^{n}(t-c)^{(n+1) q-1-v}}{\Gamma((n+1) q-v)}=\sum_{n=0}^{\infty} \frac{(a)^{n}{ }_{c} d_{t}^{u}(t-c)^{(n+1)_{q-1-v}}}{\Gamma((n+1) q-v)} .
$$

Oldham and Spanier (1974 p.67) prove the following useful form (equation (23) repeated)

$$
{ }_{a} d_{x}^{v}[x-a]^{p}=\frac{\Gamma(p+1)[x-a]^{p-v}}{\Gamma(p-v+1)} \quad p>-1,
$$

which is applied to equation (28) to yield

$$
{ }_{c} d_{t}^{u} R_{q, v}[a, c, t]=\sum_{n=0}^{\infty} \frac{(a)^{n}(t-c)^{(n+1) q-1-(v+u)}}{\Gamma((n+1) q-(v+u))} \quad q-v>0 .
$$

Thus we have the useful result

$$
{ }_{c} d_{t}^{u} R_{q, v}[a, c, t]=R_{q,(v+u)}[a, c, t] \quad q>v .
$$

That is, $u$ order differintegration of the $R$-function returns another $R$-function. 
Relationship Between $R_{\mathrm{q}, \mathrm{mq}}$ and $R_{q, 0}$

From the definition of $R$ we can write

$$
R_{q, m q}(a, c, t)=\sum_{n=0}^{\infty} \frac{(a)^{n}(t-c)^{(n+1)_{q-1-n q q}}}{\Gamma((n+1) q-m q)}=(t-c)^{-m q} \sum_{n=0}^{\infty} \frac{(a)^{n}(t-c)^{(n+1) q-1}}{\Gamma((n-m+1) q)}
$$

Letting $n-m=r$, yields

or

$$
R_{q, m q}(a, c, t)=(t-c)^{-m q} \sum_{r=-m}^{\infty} \frac{(a)^{r+m}(t-c)^{(r+m+1) q-1}}{\Gamma((r+1) q)},
$$

$$
R_{q, m q}(a, c, t)=(a)^{n} \sum_{r=0}^{\infty} \frac{(a)^{r}(t-c)^{(r+1) q-1}}{\Gamma((r+1) q)}+(a)^{m} \sum_{r=-m}^{-1} \frac{(a)^{r}(t-c)^{(r+1) q-1}}{\Gamma((r+1) q)} .
$$

Recognizing the first summation on the right hand side as $R_{4,0}(a, c, t)$ gives the final result as;

$$
R_{q, m q}(a, c, t)=(a)^{m} R_{q, 0}(a, c, t)+(a)^{m} \sum_{r=-m}^{-1} \frac{(a)^{r}(t-c)^{(r+1)_{q-1}}}{\Gamma((r+1) q)}
$$

It is noted, that when $(r+1) q \leq 0$ and integer the elements of the summation term vanish.

\section{Fractional Impulse Function}

Consider the function $R_{q, 0}(0,0, t)$, then we can write,

$$
R_{q, 0}(0,0, t)=\lim _{a \rightarrow 0} R_{q, 0}(a, 0, t)=\lim _{a \rightarrow 0} \sum_{n=0}^{\infty} \frac{(a)^{n} t^{(n+1) q-1}}{\Gamma((n+1) q)} .
$$

In the limit the terms $n>0$, of the summation vanish, thus

$$
R_{q, 0}(a, 0, t)=\lim _{a \rightarrow 0} \frac{(a)^{0} t^{q-1}}{\Gamma(q)}=\frac{t^{q-1}}{\Gamma(q)} .
$$

From equation (19) the associated Laplace transform pair is given by;

$$
L\left\{R_{q, 0}(0,0, t)\right\}=\frac{1}{s^{q}} \quad \operatorname{Re}(q)>0, \quad \operatorname{Re}(s)>0 .
$$

\section{Relationship of the $\boldsymbol{R}$-function to the Elementary Functions}

Many of the elementary functions are special cases of the $R$-function. Some of these are illustrated here.

\section{Exponential Function}

Consider $R_{1,0}(a, 0, t)$, by definition, we have

$$
R_{\mathrm{l}, 0}(a, 0, t)=\sum_{n=0}^{\infty} \frac{(a)^{n} t^{n}}{\Gamma(n+1)}=\sum_{n=0}^{\infty} \frac{(a t)^{n}}{n !},
$$

thus

$$
R_{1,0}(a, 0, t)=e^{a r}
$$


Sine Function

Consider $a R_{2,0}\left(-a^{2}, 0, t\right)$, by definition, we have

$$
a R_{2,0}\left(-a^{2}, 0, t\right)=a \sum_{n=0}^{\infty} \frac{\left(-a^{2}\right)^{n} t^{(n+1) 2-1}}{(2 n+1) !}=a\left\{t-\frac{a^{2} t^{3}}{3 !}+\frac{a^{4} t^{5}}{5 !}-\ldots\right\}
$$

thus

$$
a R_{2.0}\left(-a^{2}, 0, t\right)=\sin (a t)
$$

\section{Cosine Function}

The cosine function relates to $R_{2,1}\left(-a^{2}, 0, t\right)$, again by definition

$$
R_{2,1}\left(-a^{2}, 0, t\right)=\sum_{n=0}^{\infty} \frac{\left(-a^{2}\right)^{n} t^{(n+1) 2-1-1}}{\Gamma((n+1) 2-1)}=\sum_{n=0}^{\infty} \frac{\left(-a^{2}\right)^{n} t^{2 n}}{(2 n) !}=\left\{1-\frac{a^{2} t^{2}}{2 !}+\frac{a^{4} t^{4}}{4 !}-\ldots\right\}
$$

thus

$$
R_{2,1}\left(-a^{2}, 0, t\right)=\cos (a t)
$$

Hyperbolic Sine and Cosine

Consider $a R_{2,0}\left(a^{2}, 0, t\right)$, by definition, we have

$$
a R_{2,0}\left(a^{2}, 0, t\right)=a \sum_{n=0}^{\infty} \frac{\left(a^{2}\right)^{n} t^{(n+1) 2-1}}{\Gamma((n+1) 2)}=a \sum_{n=0}^{\infty} \frac{\left(a^{2}\right)^{n} t^{2 n+1}}{(2 n+1) !}=\left\{a t+\frac{a^{3} t^{3}}{3 !}+\frac{a^{5} t^{5}}{5 !}+\ldots\right\},
$$

thus,

In similar manner

$$
a R_{2,0}\left(a^{2}, 0, t\right)=\sinh (a t)
$$

$$
R_{2,1}\left(a^{2}, 0, t\right)=\cosh (a t)
$$

\section{$\boldsymbol{R}$-Function Identities}

Trigonometric Based Identities

A number of identities involving the $R$-function may be readily shown based on the elementary functions. The exponential function, equation (40)

$$
R_{1,0}(a, 0, x)=e^{a x},
$$

may be expressed as

$$
e^{i x}=R_{1,0}(i, 0, x)
$$

Then from equation (42)

$$
\sin (a x)=a R_{2,0}\left(-a^{2}, 0, x\right)
$$

and expressing the sine function in complex exponential terms gives

$$
\sin (x)=\frac{1}{2 i}\left(e^{i x}-e^{-i x}\right)
$$


Combining equations (49),(50) and (51) then yields the identity

$$
R_{2,0}(-1,0, x)=\frac{1}{2 i}\left(R_{1,0}(i, 0, x)-R_{1,0}(-i, 0, x)\right) \text {. }
$$

In similar manner using the cosine function, equation (44)

from which

$$
\cos (x)=R_{2,1}(-1,0, x)=\frac{1}{2}\left(e^{i x}+e^{-i x}\right)
$$

$$
R_{2,1}(-1,0, x)=\frac{1}{2}\left(R_{1,0}(i, 0, x)+R_{1,0}(-i, 0, x)\right)
$$

The hyperbolic functions may also be used as a basis, using $\sinh$ function, yields

$$
R_{2,0}(1,0, x)=\frac{1}{2}\left(R_{1.0}(1,0, x)-R_{1,0}(-1,0, x)\right)
$$

The cosh function gives

$$
R_{2,1}(1,0, x)=\frac{1}{2}\left(R_{1,0}(1,0, x)+R_{1,0}(-1,0, x)\right)
$$

Many other identities may be found based on the known trigonometric identities, a few examples follow, from

$$
\sin ^{2}(x)+\cos ^{2}(x)=1
$$

we have

$$
R_{2,0}^{2}(-1,0, x)+R_{2,1}^{2}(-1,0, x)=1 \text {. }
$$

From the identity

$$
\sin (2 x)=2 \sin (x) \cos (x)
$$

derives

$$
R_{2,0}(-1,0,2 x)=2 R_{2,0}(-1,0, x) R_{2,1}(-1,0, x)
$$

From the trigonometric identity

$$
\sin (3 x)=3 \sin (x)-4 \sin ^{3}(x)
$$

we determine the identity

$$
R_{2,0}(-1,0,3 x)=3 R_{2,0}(-1,0, x)-4 R_{2,0}^{3}(-1,0, x)
$$

Further Identities

Other identities may be derived as follows. Let $v=q-p$, then the Laplace transform of the $R$-function may be written as

$$
L\left\{R_{q, q-p}(-a, 0, t)\right\}=\frac{s^{q-p}}{s^{q}+a}=\frac{1}{s^{p-q}\left(s^{q}+a\right)} .
$$


This may be rearranged to give

$$
\frac{1}{s^{p-q}\left(s^{q}+a\right)}=\frac{1}{s^{p}}\left[1+\frac{-a}{s^{q}+a}\right] .
$$

Inverse transforming gives the identity

$$
R_{q, q-p}(-a, 0, t)=R_{p, 0}(0,0, t)-a R_{q,-p}(-a, 0, t)
$$

Another set of identities follows by factoring the denominator of Laplace transform, thus

$$
R_{q, v}(a, 0, t) \Leftrightarrow \frac{s^{v}}{s^{q}-a}=s^{v}\left[\frac{1}{\left(s^{q / 2}-a^{1 / 2}\right)\left(s^{q / 2}+a^{1 / 2}\right)}\right] .
$$

Now a partial fraction expansion of the denominator gives

$$
=s^{\nu}\left[\frac{\frac{1}{2 a^{1 / 2}}}{s^{q / 2}-a^{1 / 2}}-\frac{\frac{1}{2 a^{1 / 2}}}{s^{q / 2}+a^{1 / 2}}\right]=\left[\frac{\frac{1}{2 a^{1 / 2}} s^{v}}{s^{q / 2}-a^{1 / 2}}-\frac{\frac{1}{2 a^{1 / 2}} s^{v}}{s^{q / 2}+a^{1 / 2}}\right] .
$$

Taking the inverse transform, yields

$$
R_{q, v}(a, 0, t)=\frac{1}{2 a^{1 / 2}}\left\{R_{q / 2, v}\left(a^{1 / 2}, 0, t\right)-R_{q / 2, v}\left(-a^{1 / 2}, 0, t\right)\right\}
$$

Very many more such identities are possible, indeed because of the generality of the $R$-function, powerful meta-identities may be possible.

\section{Relationship of the $R$-Function to Other Functions}

The generality of the $R$-function allows it to be related to many other functions. In this section it will be related to the important functions discussed in the introductory section of the paper. The Laplace transform facilitates determination of the desired relationships. The double arrow will be used to indicate the transform pairs, thus for the $R$-function;

$$
R_{q, v}(a, c, t) \Leftrightarrow \frac{s^{v}}{s^{q}-a} \quad \operatorname{Re}(q-v)>0
$$

Mittag-Leffler's Function

The Mittag-Leffler function and its transform relate to the L-function as;

$$
E_{q}\left[-a t^{q}\right] \Leftrightarrow \frac{s^{q-1}}{s^{q}+a} \Leftrightarrow R_{q, q-1}(-a, 0, t)
$$

The time domain relationship is

$$
R_{q \cdot q-1}(-a, 0, t)=E_{q}\left[-a t^{q}\right]=\sum_{n=0}^{\infty} \frac{(-a)^{n} t^{n q}}{\Gamma(n q+1)} .
$$

Also, because ${ }_{c} d_{t}^{\alpha} R_{q, v}[-a, c, t]=R_{q \cdot(v+\alpha)}[-a, c, t]$ it follows that

$$
{ }_{c} d_{t}^{q-1} R_{q .0}(-a, c, t)=E_{q}\left[-a(t-c)^{q}\right]
$$




\section{Argarwal's Function}

The Argarwal function and its transform relate to the $R$-function as follows;

$$
E_{q, p}\left[t^{q}\right] \Leftrightarrow \frac{s^{q-p}}{s^{q}-1} \Leftrightarrow R_{q, q-p}(1,0, t) .
$$

The time domain relationship is

$$
R_{q, q-p}(1,0, t)=E_{q, p}\left[t^{q}\right]=\sum_{n=0}^{\infty} \frac{t^{n q-1+p}}{\Gamma(n q+p)}
$$

\section{Erdelyi's Function}

The relationship between the Erdelyi function and the $R$-function is given by

$$
R_{q, q-\beta}(1,0, t)=t^{1-\beta} E_{q, \beta}\left[t^{q}\right]=t^{1-\beta} \sum_{n=0}^{\infty} \frac{t^{n q}}{\Gamma(n q+\beta)} .
$$

\section{Robotnov and Hartley Function}

The F-function and its transform relate to the $R$-function as follows;

$$
F_{q}[-a, t] \Leftrightarrow \frac{1}{s^{q}+a} \Leftrightarrow R_{q, 0}(-a, 0, t) .
$$

The time series common to these functions is given as;

$$
R_{q, 0}(-a, 0, t)=F_{q}[-a, t]=\sum_{n=0}^{\infty} \frac{(-a)^{n} t^{(n+1) q-1}}{\Gamma((n+1) q)} .
$$

Miller and Ross's Function

The Miller and Ross function and its transform relate to the $R$-function as follows

$$
E_{t}(v, a) \Leftrightarrow \frac{s^{-v}}{s-a} \Leftrightarrow R_{\mathrm{l},-v}(a, 0, t) .
$$

The time series common to these functions is given as;

$$
R_{1,-v}(a, 0, t)=E_{l}(v, a)=\sum_{n=0}^{\infty} \frac{(a)^{n} t^{n+v}}{\Gamma(n+v+1)} .
$$

\section{Example - The Dynamic Thermocouple}

This problem was introduced originally in Lorenzo and Hartley 1998, and frequency domain solutions are presented there. Here, it is desired to determine the time domain dynamic response of the thermocouple, figure 4 , which is designed to achieve rapid response. The thermocouple consists of two dissimilar metals with a common junction point. To achieve a high level of dynamic response, the mass of the junction and the diameter of the wire are minimized. Because the wires are long and insulated they will be treated as semi-infinite (heat) conductors. This analysis will determine the time response of the junction temperature $T_{b}(s)$ in response to the

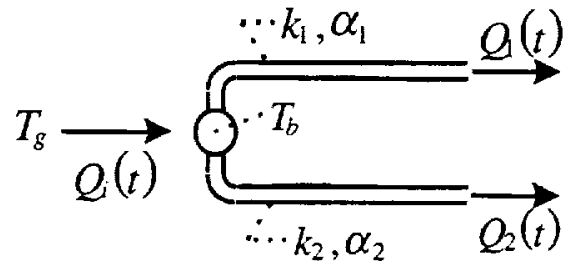

Figure 4. Dynamic Thermocouple 
free stream gas temperature $T_{g}(s)$. For the semi-infinite conductors the conducted heat rate $Q(t)$ is given by

$$
Q_{j}(t)=\frac{k_{j}}{\sqrt{\alpha_{j}}} c_{t}^{1 / 2} T_{b},
$$

where $k$ is the thermal conductivity and $\alpha$ is the thermal diffusivity. For the transfer function the effects of initialization are not required, therefore, all $\psi(t)$ 's are zero. Thus the following equations describe the time domain behavior:

$$
\begin{gathered}
Q_{i}(t)=h A\left(T_{g}(t)-T_{b}(t)\right), \\
T_{b}(t)=\frac{1}{w c_{v}}{ }_{0} D_{t}^{-1}\left(Q_{i}(t)-Q_{1}(t)-Q_{2}(t)\right) \\
Q_{1}(t)=\frac{k_{1}}{\sqrt{\alpha_{1}}}{ }_{0} D_{t}^{1 / 2} T_{b}(t)=\frac{k_{1}}{\sqrt{\alpha_{1}}}\left({ }_{0} d_{t}^{1 / 2} T_{b}(t)+\psi_{1}\left(T_{b}, 1 / 2, a, 0, t\right)\right) \text { and } \\
Q_{2}(t)=\frac{k_{2}}{\sqrt{\alpha_{2}}}{ }_{0} D_{t}^{1 / 2} T_{b}(t)=\frac{k_{2}}{\sqrt{\alpha_{2}}}\left({ }_{0} d_{t}^{1 / 2} T_{b}(t)+\psi_{2}\left(T_{b}, 1 / 2, a, 0, t\right)\right)
\end{gathered}
$$

where $h A$ is the product of the convection heat transfer coefficient and the surface area and $w c_{v}$ is the product of the junction mass and the specific heat of the material. Taking the Laplace transform of these equations yields

$$
\begin{array}{r}
Q_{i}(s)=h A\left(T_{s}(s)-T_{b}(s)\right) \\
T_{b}(s)=\frac{1}{w c_{v}}\left(\frac{1}{s}\left[Q_{i}(s)-Q_{1}(s)-Q_{2}(s)\right]+\psi_{3}(s)\right) \\
Q_{1}(s)=\frac{k_{1}}{\sqrt{\alpha_{1}}}\left(s^{1 / 2} T_{b}(s)+\psi_{1}(s)\right) \\
Q_{2}(s)=\frac{k_{2}}{\sqrt{\alpha_{2}}}\left(s^{1 / 2} T_{b}(s)+\psi_{2}(s)\right)
\end{array}
$$

Eliminating the $Q$ 's, and solving for $T_{b}(s)$ yields

$$
T_{b}(s)=\left(\frac{\frac{1}{w c_{v}}}{s+b s^{1 / 2}+c}\right)\left\{h A T_{g}(s)-\frac{k_{1}}{\sqrt{\alpha_{1}}} \psi_{1}(s)-\frac{k_{2}}{\sqrt{\alpha_{2}}} \psi_{2}(s)+s \psi_{3}(s)\right\}
$$

where $b=\frac{1}{w c_{v}}\left\{\frac{k_{1}}{\sqrt{\alpha_{1}}}+\frac{k_{2}}{\sqrt{\alpha_{2}}}\right\}$, and $c=\frac{h A}{w c_{r}}$. Factoring the leading denominator and 
expanding in partial fractions gives

$$
T_{b}(s)=\frac{1}{w c_{v}}\left(\frac{\frac{1}{\beta_{2}-\beta_{1}}}{s^{1 / 2}+\beta_{1}}+\frac{\frac{1}{\beta_{1}-\beta_{2}}}{s^{1 / 2}+\beta_{2}}\right)\left\{h A T_{g}(s)-\frac{k_{1}}{\sqrt{\alpha_{1}}} \psi_{1}(s)-\frac{k_{2}}{\sqrt{\alpha_{2}}} \psi_{2}(s)+s \psi_{3}(s)\right\},
$$

where $\beta_{1}=\frac{b}{2}+\frac{1}{2} \sqrt{b^{2}-4 c}$ and $\beta_{2}=\frac{b}{2}-\frac{1}{2} \sqrt{b^{2}-4 c}$. Then with appropriate choices for the functions of $s$ in the right most bracket this equation may be inverse transformed to yield the time domain response. To demonstrate the value of the $R$-function, we select (determine)

$\psi_{3}(s)=T_{b}(0) / s$, Further assume $T_{g}(t)=2 T_{b}(0)+t \Rightarrow T_{g}(s)=\frac{2 T_{b}(0)}{s}+\frac{1}{s^{2}}$, and $\psi_{1}(s)=\psi_{2}(s)$ are arbitrary functions of time. The solution may be written directly as:

$$
\begin{aligned}
& T_{b}(t)= \frac{h A}{w c_{v}\left(\beta_{2}-\beta_{1}\right)}\left[2 T_{b}(0) R_{1 / 2,-1}\left(-\beta_{1}, 0, t\right)+R_{1 / 2,-2}\left(-\beta_{1}, 0, t\right)\right. \\
&\left.-2 T_{b}(0) R_{1 / 2,-1}\left(-\beta_{2}, 0, t\right)-R_{1 / 2,-2}\left(-\beta_{2}, 0, t\right)\right]- \\
& \frac{1}{w c_{v}\left(\beta_{2}-\beta_{1}\right)}\left(\frac{k_{1}}{\sqrt{\alpha_{1}}}+\frac{k_{2}}{\sqrt{\alpha_{2}}}\right) \int_{0}^{t}\left\{R_{1 / 2,0}\left(-\beta_{1}, 0, t-\tau\right)-R_{1 / 2,0}\left(-\beta_{2}, 0, t-\tau\right)\right\}_{1}(\tau) d \tau+ \\
& \quad \frac{1}{w c_{v}\left(\beta_{2}-\beta_{1}\right)} T_{b}(0)\left\{R_{1 / 2,0}\left(-\beta_{1}, 0, t\right)-R_{1 / 2,0}\left(-\beta_{2}, 0, t\right)\right\} .
\end{aligned}
$$

\section{Further Generalized Functions}

Functions yet more general than the $R$-function may be developed. One such function will be derived here. It is simpler here to work backward from the s-domain to the time domain. Thus, we consider the following function

$$
G(s)=\frac{s^{v}}{\left(s^{q}-a\right)^{r}}
$$

where $v, q$, and $r$ are not constrained to be integers. Then this may be written as

$$
G(s)=s^{v-q r}\left(1-\frac{a}{s^{q}}\right)^{-r} \text {. }
$$

Now the parenthetical expression may be expanded using the binomial theorem to give

$$
G(s)=s^{v-q r} \sum_{j=0}^{\infty} \frac{\Gamma(1-r)}{\Gamma(1+j) \Gamma(1-j-r)}\left(\frac{-a}{s^{q}}\right)^{j}, \quad\left|\frac{a}{s^{q}}\right|<1,
$$

or

$$
G(s)=\sum_{j=0}^{\infty} \frac{\Gamma(1-r)}{\Gamma(1+j) \Gamma(1-j-r)}(-a)^{j} s^{v-q r-q j} .
$$


This expression may be term by term inverse transformed yielding

$$
G_{q, v, r}[a, t]=\sum_{j=0}^{\infty} \frac{\Gamma(1-r)(-a)^{j} t^{(r+j) q-v-1}}{\Gamma(1+j) \Gamma(1-j-r) \Gamma((r+j) q-v)}, \operatorname{Re}\left(q r-v>0, \operatorname{Re}(s) 0,\left|\frac{a}{s^{q}}\right|<1 .\right.
$$

Thus we have the following transform pair

$$
L\left\{G_{q, v, r}[a, t]\right\}=\frac{s^{v}}{\left(s^{q}-a\right)}, \quad \operatorname{Re}(q r-v)>0, \operatorname{Re}(s)>0,\left|\frac{a}{s^{q}}\right|>0 .
$$

The form of equation (96) presents evaluation difficulties, since when $r$ is an integer $\Gamma(1-r)$ and $\Gamma(1-j-r)$ can become infinite. Equation (96) maybe rewritten as follows: from Spanier and Oldham (p.414, eq.43:5:5)

$$
\Gamma(x-n)=\frac{\Gamma(x)}{(x-1)(x-2) \cdots(x-n)}=\frac{(-1)^{n} \Gamma(x)}{(1-x)_{n}} \quad n=0,1,2, \cdots
$$

where $(1-x)_{n}$ is the Pochhammer polynomial. From this result with $x=1-r$, we can write

$$
\Gamma(1-j-r)=\frac{\Gamma(1-r)}{(-r)(-1-r) \cdots(1-r-j)}=\frac{(-1)^{j} \Gamma(1-r)}{(r)_{j}} \quad j=1,2, \cdots
$$

Substituting this result in equation (96), yields the following more computable results

$$
G_{q, v, r}[a, t]=\sum_{j=0}^{\infty} \frac{\{(-r)(-1-r) \cdots(1-j-r)\}(-a)^{j} t^{(r+j) q-v-1}}{\Gamma(1+j) \Gamma((r+j) q-v)} .
$$

or in terms of the Pochhammer polynomial

$$
G_{q, v, r}[a, t]=\sum_{j=0}^{\infty} \frac{(r)_{j}(a)^{j} t^{(r+j) q-v-1}}{\Gamma(1+j) \Gamma((r+j) q-v)}, \operatorname{Re}(q r-v)>0, \operatorname{Re}(s)>0,\left|\frac{a}{s^{q}}\right|<1 .
$$

In similar manner relationships of increasing generality may be determined. Podlubny (1999) presents a form that is a special case of the $G$-function where $r$ is constrained to be an integer. It is also clear that taking $r=1$ specializes the $G$-function into the $R$-function. It is the authors' judgment that the $F$ - and $R$-functions will prove to be the most useful in practical applications. Table 1 summarizes the advanced functions studied in this paper along with their defining series and Laplace Transforms. 
Table 1 Summary of Defining Series and Laplace

\begin{tabular}{|c|c|c|c|}
\hline Function & Time Expression & Laplace Transform & Remarks \\
\hline Mittag-Leffler & $E_{q}\left[a t^{q}\right]=\sum_{n=0}^{\infty} \frac{a^{n} t^{n q}}{\Gamma(n q+1)}$ & $\frac{s^{q}}{s\left(s^{q}-a\right)}$ & $(q-1)_{\text {differintegral of }}$ \\
\hline Agarwal & $E_{\alpha, \beta}\left(t^{q}\right)=\sum_{m=0}^{\infty} \frac{t^{\left(m+(\beta-1)^{\alpha} \alpha\right)_{z}}}{\Gamma(\alpha m+\beta)}$ & $\frac{s^{\alpha-\beta}}{s^{\alpha}-1}$ & \\
\hline Erdelyi & $E_{\alpha, \beta}(t)=\sum_{m=1}^{\infty} \frac{t^{m}}{\Gamma(\alpha m+\beta)}$ & $\sum_{m=0}^{\infty} \frac{\Gamma(m+1)}{\Gamma(c m+\beta) b^{m+1}}$ & $\alpha, \beta>0$ \\
\hline Robotnov / Hartley & $F_{q}[a, t]=\sum_{n=0}^{\infty} \frac{a^{n} t^{(n+1))_{q-1}}}{\Gamma((n+1) q)}$ & $\frac{1}{s^{q}-a}$ & eigenfunction \\
\hline Miller-Ross & $E_{r}(v, a)=\sum_{k=0}^{\infty} \frac{a^{k} t^{k+v}}{\Gamma(v+k+1)}$ & $\frac{s^{-v}}{s-a}$ & \\
\hline Current Paper & $R_{q, v}(a, t)=\sum_{n=0}^{\infty} \frac{a^{n} t^{(n+1) k_{n-1-v}}}{\Gamma((n+1) q-v)}$ & $\frac{s^{v}}{s^{4}-a}$ & eigenfunction \& differintegral \\
\hline Current Paper & $G_{q, v, r}(a, t)=\sum_{i=0}^{\infty} \frac{(r)_{j}(-a)^{i} t^{\left(\left(r+j k_{k-1-v)}\right)\right.}}{(-1)^{i} \Gamma(1+j) \Gamma((r+i) q-v)}$ & $\frac{s^{v}}{\left(s^{q}-a\right)^{r}}$ & $\begin{array}{l}\text { eigenfunction \& differintegrals } \\
\text { repeated \& partially rep. }\end{array}$ \\
\hline
\end{tabular}

\section{Summary}

This paper has presented a new function for use in the fractional calculus, it is called the $R$-function. The $R$-function is unique in that it contains all of the derivatives and integrals of the F-function. The $R$-function has the eigen-property, that is it returns itself on $q$ th order differintegration. Special cases of the $R$-function also include the exponential function, the sine, cosine, hyperbolic sine and hyperbolic cosine functions. Further, the $R$-function contains, as special cases; the Mittag-Leffler function, Agarwal's function, Erdelyi's function, Hartley's F-function, and Miller and Ross's function. Numerous identities are possible with the $R$-function some of these have been shown in the text.

The value of the $R$-function is clearly demonstrated in the dynamic thermocouple problem where it enables the analyst to directly inverse transform the Laplace domain solution, (operational $(s)$ form) to obtain the time domain solution.

A further generalization of the $R$-function, called the $G$-function brings in the effects of repeated and partially repeated fractional poles. This generalization carries increased time domain complexity.

A $R$-function based trigonometry is also possible. It is a generalization of the conventional trigonometry, and will be the subject of a future paper. 


\section{References}

Erdelyi, A., Editor, Magnus, W., Oberhetinger F., and Tricomi F.G., Tables of Integral Transforms, vol. 1, McGraw-Hill Book Co., 1954, LCC Number 54-6214.

Hartley, T.T. and Lorenzo, C.F., A Solution to the Fundamental Linear Fractional Order Differential Equation, NASA /TP_1998-208963, December 1998.

Hartley, T.T. and Lorenzo, C.F., The Vector Linear Fractional Initialization Problem, NASA TP-1999-208919, May 1999.

Miller, K.S. and Ross, B., An Introduction to the Fractional Calculus and Fractional Differential Equations, John Wiley \& Sons, Inc., 1993.

Mittag-Leffler, M.G., Une generalisation de l'integrale de Laplace-Abel, Proc. Paris Academy of Science, pp. 537-539, March 2, 1903(a).

Mittag-Leffler, M.G., Sur la nouvelle fontion $E_{a}(x)$, Proc. Paris Academy of Science, pp. 554-558, October 21, 1903(b).

Mittag-Leffler, M.G., Sur la representation analytique d'une branche uniforme d'une fonction monogene, Acta Mathematica, vol. 29, pp. 101-181, 1905.

Podlubny, I., Fractional Order Systems and $P I^{2} D^{n}$ Controllers, IEEE Transactions on Automatic Control, vol. 44, no. 1, January 1999.

Spanier, J. and Oldham, K.B., An Atlas of Functions, Hemisphere Publishing Corp. (Subsidiary of Harper \& Row, Publishers Inc.) 1987.

Wylie, C.R., Advanced Engineering Mathematics, Fourth Edition, McGraw-Hill Book Co., 1975.

Robotnov, Y.N., Tables of a Fractional Exponential Function of Negative Parameters and Its Integral (in Russian), Nauka, Russia (1969).

Robotnov, Y.N., Elements of Hereditary Solid Mechanics (in English) MIR Publishers, Moscow, 1980. 


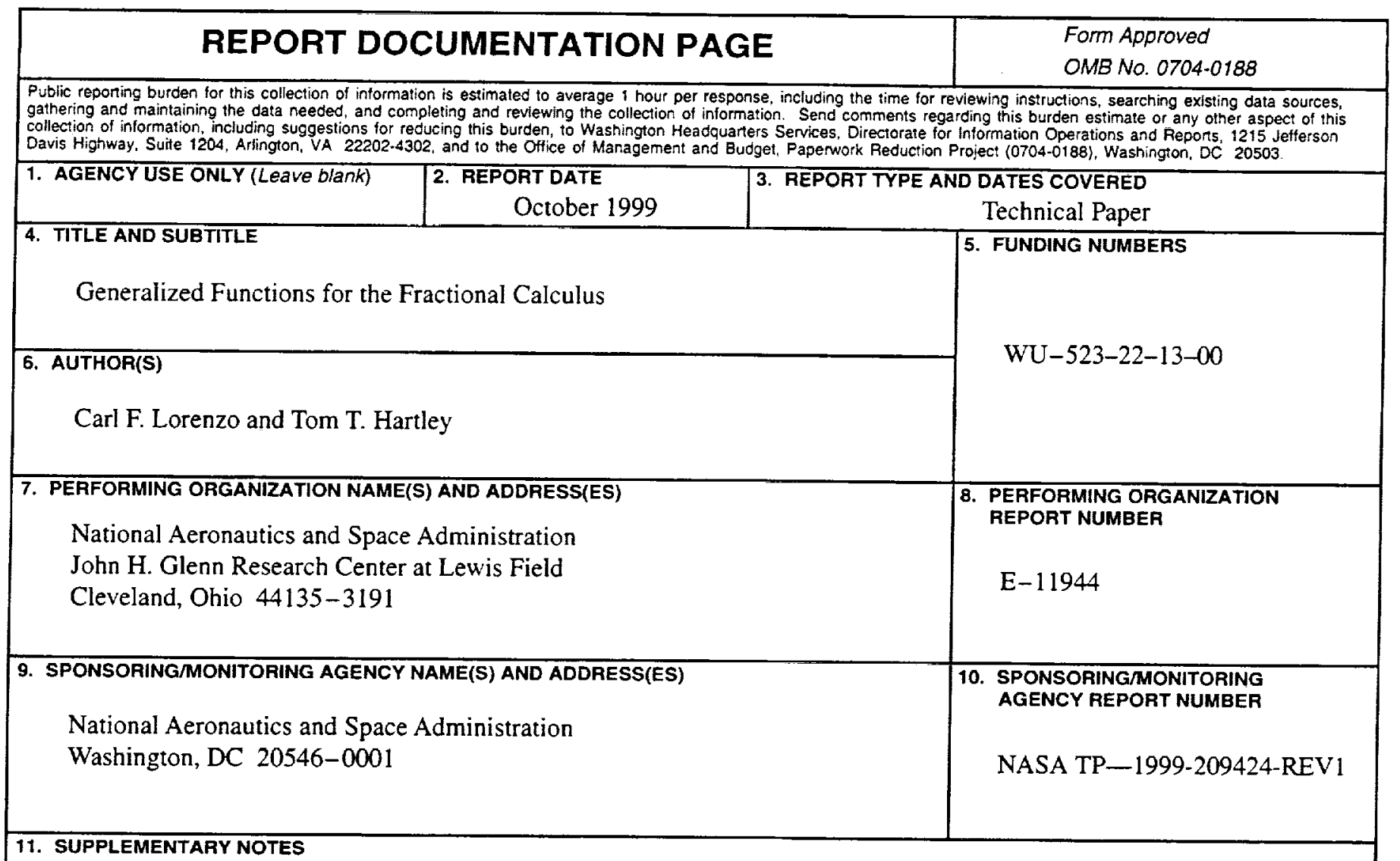

Carl F. Lorenzo, NASA Glenn Research Center, and Tom T. Hartley, The University of Akron, Department of Electrical Engineering, Akron, Ohio 44325-3904. Responsible person, Carl F. Lorenzo, organization code 5500, (216) 433-3733.

12a. DISTRIBUTION/AVALABILITY STATEMENT

12b. DISTRIBUTION CODE

Unclassified - Unlimited

Subject Categories: 59, 66, and $67 \quad$ Distribution: Standard

This publication is available from the NASA Center for AeroSpace Information, (301) 621-0390.

13. ABSTRACT (Maximum 200 words)

Previous papers have used two important functions for the solution of fractional order differential equations, the MittagLeffler function $E_{q}\left[a t^{q}\right]$ (1903a, 1903b, 1905), and the F-function $F_{q}[a, t]$ of Hartley \& Lorenzo (1998). These functions provided direct solution and important understanding for the fundamental linear fractional order differential equation and for the related initial value problem (Hartley and Lorenzo, 1999). This paper examines related functions and their Laplace transforms. Presented for consideration are two generalized functions, the $R$-function and the $G$-function, useful in analysis and as a basis for computation in the fractional calculus. The $R$-function is unique in that it contains all of the derivatives and integrals of the F-function. The $R$-function also returns itself on $q$ th order differ-integration. An example application of the $R$-function is provided. A further generalization of the $R$-function, called the $G$-function brings in the effects of repeated and partially repeated fractional poles.

14. SUBJECT TERMS

Fractional calculus; Fractional differential equations; Systems; Generalized functions; Eigenfunctions

\begin{tabular}{|c|c|c|}
\hline $\begin{array}{c}\text { 17. SECURITY CLASSIFICATION } \\
\text { OF REPORT } \\
\text { Unclassified }\end{array}$ & $\begin{array}{c}\text { 18. SECURITY CLASSIFICATION } \\
\text { OF THIS PAGE } \\
\text { Unclassified }\end{array}$ & $\begin{array}{c}\text { 19. SECURITY CLASSIFICATION } \\
\text { OF ABSTRACT } \\
\text { Unclassified }\end{array}$ \\
\hline
\end{tabular}

